$\underline{\text { Review Article }}$

\title{
PORPHYSOMES-A PARADIGM SHIFT IN TARGETED DRUG DELIVERY
}

\author{
REVATHY B. MENONa, LAKSHMI V. S. a, AISWARYA M. U., KEERTHANA RAJUa, SREEJA C. NAIR ${ }^{{ }^{*}}$ \\ aDepartment of Pharmaceutics, Amrita School of Pharmacy, Amrita Vishwa Vidyapeetham, AIMS Health Science Campus, Kochi, India \\ Email: sreejacnair@aims.amrita.edu
}

Received: 04 Nov 2017, Revised and Accepted: 09 Jan 2018

\section{ABSTRACT}

A novel drug delivery system is the one that ensures optimum dose at the right time, at the right location. Porphysomes are among those drug delivery systems. Porphysomes are a means of vesicular drug delivery systems. They are liposome-like structures composed completely of porphyrin lipid. The porphysomes encapsulates the active medicament in vesicular structure. They are having an aqueous core which can be loaded with the medicament. They have the capacity to destroy the disease tissues. They absorb the heat in the near infrared region and release this heat to destroy the diseased tissues. Porphysomes are having immense applications in the field of positron-electron therapy (PET), photoacoustic imaging, photothermal therapy etc. This review article discusses regarding the Porphysome-the drug delivery system, its advantages and disadvantages, composition, method of preparation, applications and various aspects related to the porphysomal drug delivery.

Keywords: Porphyrin, Phospholipid bilayer, Vesicular drug delivery, Imaging, Biophotonic property

(c) 2018 The Authors. Published by Innovare Academic Sciences Pvt Ltd. This is an open access article under the CC BY license (http://creativecommons.org/licenses/by/4.0/] DOI: http://dx.doi.org/10.22159/ijap.2018v10i2.23493

\section{INTRODUCTION}

In the past few years, a great focus has been laid on the development of different novel drug delivery systems (NDDS) [1-5]. A novel drug delivery system is the one that ensures optimum dose at the right time and at the right location. It brings together approaches, formulations, technologies for transporting a drug in the body in order to exhibit its desired therapeutic effects [6-10]. For the last few years, many NDDS has come in to use such as liposomes, sphingosomes, niosomes etc. But now the NDDS is towards a new discovery, the porphysomes.

The porphysomes are a means of vesicular drug delivery systems. They encapsulate the active medicament in a vesicular structure [11]. They are liposome-like structures composed completely of porphyrin lipid. Porphysomes are bilayered spherical nano-sized vesicles formed from the self-assembled porphyrins. Each porphyrin is having a diameter of $100 \mathrm{~nm}$. Like liposomes, porphysomes are having an aqueous core that can be loaded with therapeutic drugs [12]. These vesicular systems enhance the existence of drugs in systemic circulation and thereby decreases toxicity. It possesses multimodal biophotonic properties. They are used in photoacoustic imaging, positron emission tomography (PET), magnetic resonance imaging (MRI) and image tracked drug delivery. Porphysomes are phototherapy agents. They have the capacity to destroy the diseased tissues. They absorb the light in the near-infrared region and then releases this heat to destroy the diseased tissues.

Many forms of inorganic compounds were used for thermal phototherapy and photoacoustic imaging because these compounds are having high biophotonic properties. But these inorganic compounds are non-biodegradable and also have long-term toxicity. All these challenges were overcome by the introduction of novel colloidal drug delivery system called porphysomes. They possess strong biophotonic properties and are completely composed of organic porphyrin-lipid units.

\section{Novel drug delivery systems}

A novel drug delivery system is a system that helps in maintaining the concentration of drug in therapeutically effective range. Some drugs exhibit their maximum effect within an optimum concentration range. Either no therapeutic activity is produced by the drug below this optimum concentration or toxicity is produced above this concentration [13].

\section{Controlled drug delivery systems}

Controlled drug delivery system is a mode of drug delivery that ensures uniform concentration of drug to the site of absorption. Due to this property, it maintains plasma concentration within the therapeutic range, as in fig. 1 [14]. It helps to reduce the frequency of administration. It also minimizes the toxicity caused by drugs.
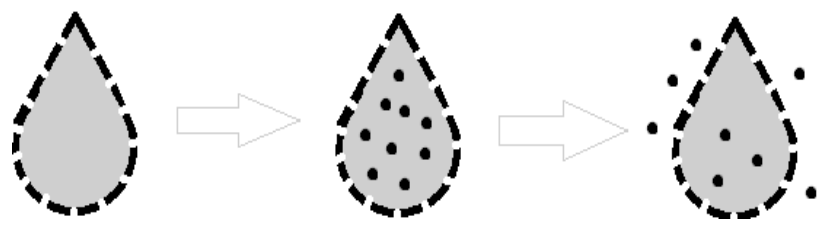

Fig. 1: Controlled drug delivery system for drug delivery

\section{Carriers used in targeted drug delivery}

Carriers which are also called as vehicles or drug vectors are the most important component of a targeted drug delivery system that helps in the successful transportation of the drug to the specified sites.

\section{Characteristics of an ideal carrier}

- It should be able to cross the blood-brain barrier (BBB)

- It should maintain the specificity of the surface ligands

- It should be specifically recognized by the target cells

- A stable drug-ligand complex must be formed

- The carriers used should be biodegradable, non-toxic and nonimmunogenic in nature

- The carrier systems after recognizing the target sites should release the drugs inside the specific organs, tissues or cells [15].

\section{Porphysomes as a lipoidal drug carrier}

Among lipoidal biocarriers 'Porphysomes' are the emerging drug delivery systems under study. As discussed earlier, they are liposome-like structures composed completely of porphyrin lipid. They possess a lipoidal bilayered structure, encapsulating the active ingredient in the central core. Porphysomes comprise of a phospholipid bilayer and possess a porphyrin ring [16].

\section{Composition of porphysomes}

Porphysomes are composed of porphyrin and phospholipid. 


\section{Porphyrin}

Porphyrins are the building blocks of porphysomes. They are heterocyclic organic compounds which are made up of four pyrrole subunits which are interconnected by methane bridges at their $\alpha$ carbon as depicted in fig. 2 [17]. The porphyrin is having 26 electrons in its conjugated system.

They mostly occur naturally in pigments like haemoglobin (heme), chlorophyll in plants etc.

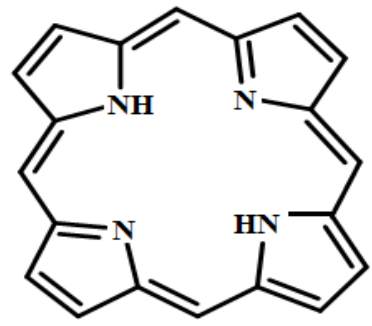

Fig. 2: Porphyrin

\section{Phospholipids}

Phospholipids are major components of cell membranes. They are mainly composed of two hydrophobic fatty acid tails and hydrophilic heads consisting of phosphate group as shown in fig. 3 [18]. They are amphiphilic in nature, hence can form bilayers $[19,20]$.

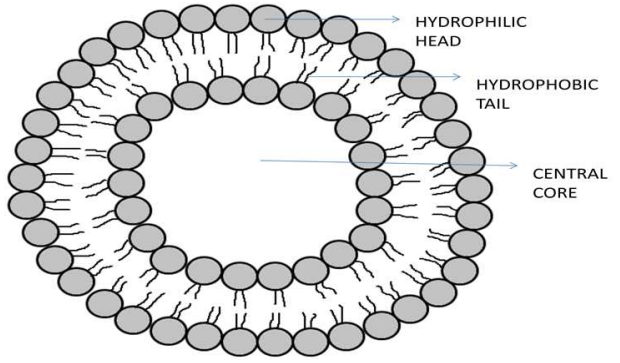

Fig. 3: Phospholipid bilayer

There are different types of phospholipids used based on the changes in the aliphatic chain, head groups and alcoholic parts. Some of those examples are;

$>$ Phosphotidyl Choline (Lecithin)

$>$ Phosphotidyl Ethanolamine (Cephalin)

> Phosphotidyl Serine

$>$ Phosphotidyl Inositol [21, 22].

Advantages and disadvantages of porphysomes [23-26]

The porphysomes have a large number of advantages when compared with other forms of drug delivery systems. Along with advantages, these are also having disadvantages too. The advantages and disadvantages are given in table 1.

Table 1: Advantages and disadvantages of porphysomes

\begin{tabular}{ll}
\hline Advantages of porphysomes & Disadvantages of porphysomes \\
\hline 1) Increases bioavailability of drugs & 1) Encapsulated drugs are viable to leakage \\
2) Decreases toxicity effect of certain drugs & 2) Less stability \\
3) Increases the stability of drugs via encapsulation & 3) Short half-life \\
4) Reduce exposure of sensitive tissues to toxic drugs & 4) High production cost \\
5) Increases circulation lifetime of drugs & \\
6) Delays the elimination of rapidly metabolisable drugs & \\
7) Completely biodegradable & \\
8) Easily modifiable & \\
\hline
\end{tabular}

\section{Preparation}

Porphysome nanovescicles are formed by the self-assembly of 'porpholipids'. By chemically fusing porphyrin molecules with phospholipids, porpholipids are created. These porpholipids spontaneously assembles into bilayers, or lipid sheets, when these are suspended in water and heated.

Then these bilayers can be forced out through a tiny perforated membrane to form balloon-like nanoparaticle with a diameter of $100 \mathrm{~nm}$, which are termed as porphysomes, as depicted fig. 4 [27].

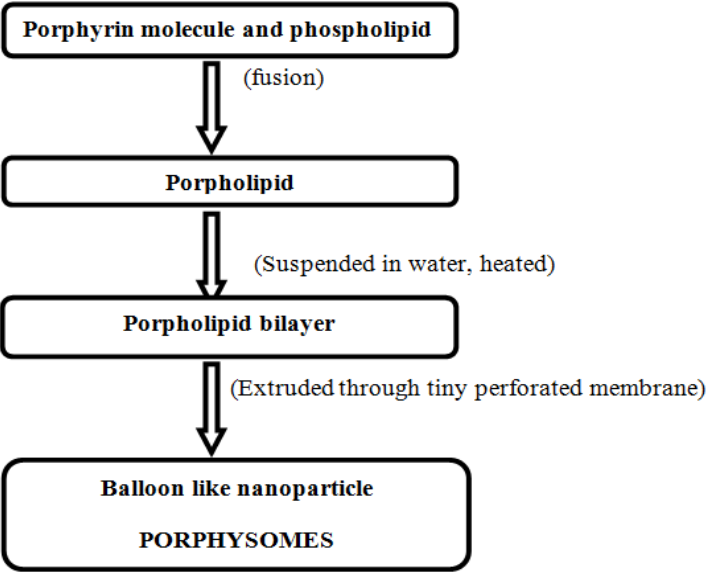

Fig. 4: Preparation of porphysomes

\section{Properties of porphysomes}

The porphysomes possess a large number of properties. Some of the properties are given below:

1) Photothermal therapy

2) Photodynamic therapy

3) Photoacoustic imaging

4) Exploitation of stable $\mathrm{Cu}^{2+}$

5) Radiolabelling

6) Liposomal properties

\section{1) Photothermal therapy}

Photothermal therapy is one of the latest developed therapeutic strategy, that uses the near-infrared (NIR) laser photoabsorbers,which upon NIR laser irradiation generate heat and thermally ablate the cancer cells. Phototherml therapy are very advantageous in cancer therapy. They exhibit high specificity, minimal invasiveness, precise temporal selectivity. They directly eradicate cancer cells in the primary tumor. The complete information regarding the photothermal therapy is explained in Jilon Wang et al. [28, 29].

\section{2) Photodynamic therapy}

Photodynamic therapy is a type of treatment which uses photosensitisers for the destruction of cancerous cells. It is a minimal invasive mode of treatment. Photosensitisers have an ability to produce a biochemical reaction by absorbing the light [30]. The photosensitizers used initiates a cytotoxic activity towards malignant cancerous cells. The cytotoxic activity is due to the generation of 'singlet oxygen'. 
The photosensitisers used in photodynamic therapy are mostly tetrapyrrole derivatives, like protoporphyrins [31].

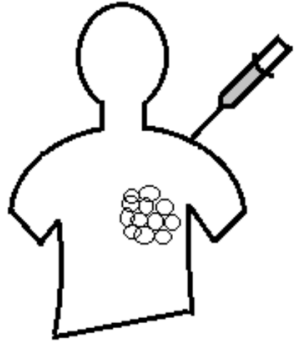

a)Chemical is Injected into the body.

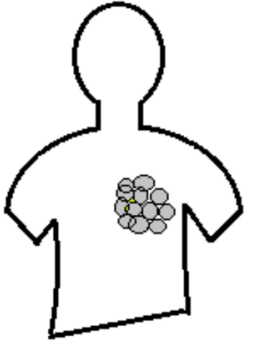

b)The chemical gets concentrated at the Tumor site.
A schematic diagram depicted in Januz B et al., explain the different steps involved in photodynamic therapy are depicted in the fig. fig. 5 [32] below:

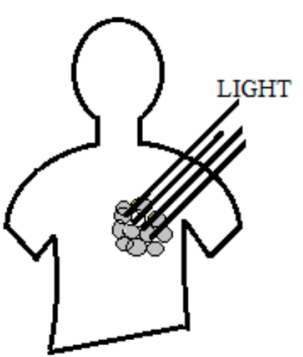

c) The chemical is activated by the Light.

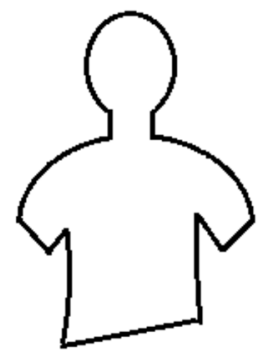

d)The tumor cells get destroyed.

Fig. 5: Photodynamic therapy

\section{3) Photoacoustic imaging}

The photoacoustic imaging refers to the generation of acoustic waves, by absorbing the electromagnetic energy [33]. The radio frequency (rf) pulses or short pulses, which are non-ionising waves, are used to excite the megahertz ultrasound waves, referred to as photoacoustic or thermoacoustic signals, in biological tissues. Nonionizing waves are used upon ionising waves because the former does not cause any health hazards. The general steps involved in the process of photoacoustic imaging is depicted below in fig. 6 [34].

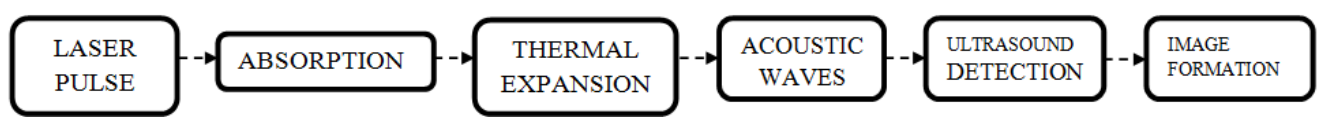

Fig. 6: Steps in photoacoustic imaging

\section{4) Radiolabelling}

Radiolabeling is a technique used for tracking the passage of a sample substance through a biological system. Radionucleotides are used to label the substance, in its chemical composition. These radionucleotides gets decayed, and emit minute amounts of radioactivity. This helps in tracking their presence in the body. Radiolabeling is having a critical importance in drug development process [35].

\section{5) Liposomal properties}

Liposomes are one of the vesicular, targeted drug delivery systems. These are spherical vesicles consisting of one or more phospholipid bilayers [36]. These systems are having both hydrophobic and hydrophilic characters. Their size ranges from $30 \mathrm{~nm}$ to several nanometers[37,38]. They are highly biocompatible, non-toxic, biodegradable. Due to all these properties, liposomes are used as carriers of both hydrophilic andn lipophilic drugs.

Porphysomes possess all these properties as that of liposomes. Porphysomes are also vesicular, made of a phospholipid bilayer, biodegradable etc. As dicussed earlier porphysomes are having large aqueous core which can be loaded with drugs. This is also another property that resembles liposomes.

\section{Compounds with porphyrin ring}

\section{1) Hemoglobin}

Hemoglobin is a protein molecule that is present in the red blood cells [RBC] which is made up of two parts: heme and globin. It is made up of four protein molecules that are connected together.

Heme part of hemoglobin is a porphyrin compound which carries an iron group as shown in fig. 7 [39]. The centre of porphyrin ring is occupied by the iron. This iron is linked to all the four nitrogen atoms of all pyrrole rings of porphyrin. It is also linked to the nitrogen of imidazole ring present in the globin part i. e to the histidine. Four polypeptide chains, two $\alpha$ and two $\beta$ chains, constitute the globin part. $\alpha_{2} \beta_{2}$ forms the normal adult hemoglobin. Their main function in mammals is to transport $\mathrm{O}_{2}$, from lungs to tissues [40].

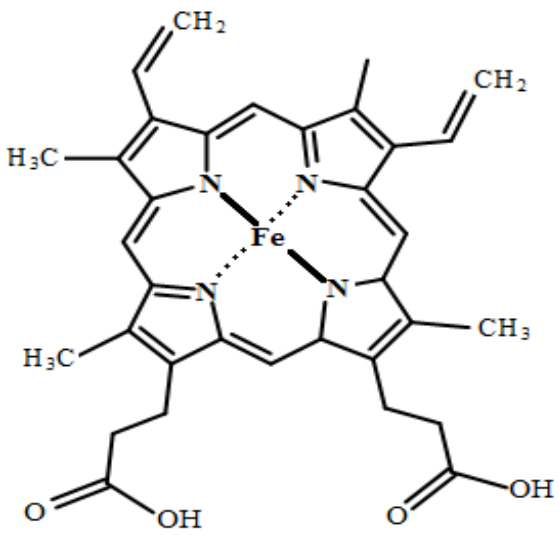

Fig. 7: Structure of hemoglobin

\section{2) Chlorophyll}

Chlorophyll is a green colour pigment seen in the leaves and stems of green plants. It is a chemical compound that helps the plants to make energy by capturing the sunlight. It gives plants the green colour [41]. The basic structure of chlorophyll is the porphyrin ring with a central atom, a metal atom. Here the central metal atom is magnesium. The central metal atom is bonded to the nitrogen atoms of the four pyrrole rings of the porphyrin ring. 
The chlorophyll is a chemoprotective agent. It has an abiliy to prevent the absorption of toxins. It also possesses anti-microbial properties [42].

\section{3) Myoglobin}

Myoglobin is another pigment/molecule that carries a porphyrin ring. It is having a similar structure as that of hemoglobin. It gets its name as it possess same structure as well as functions as hemoglobin. It is a hemoprotein present in cytoplasm. Like hemoglobin, myoglobin binds with $\mathrm{O}_{2}$ and also plays a major role in the transport of oxygen from RBC to mitochondria. It is a oxygen storage protein in the muscles [43]. It is primarily seen in cardiomyocytes and oxidative skeletal muscles. It helps in the nitric oxide homeostasis in skeletal muscles [44-46].

\section{4) Cobalamine}

Cobalamine is also called as 'vitamin $\mathrm{B}_{12}$ '. It is a compound consisting of a tetrapyrrole ring with cobalt as the central metal atom. Such a structure is called as corrin ring. It is a similar structure as that of the porphyrin ring. A small difference is that the corrin ring lacks a carbon when compared to porphyrin as shown fig. 8 [47].

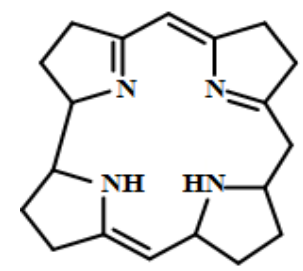

Fig. 8: Corrin ring

\section{5) Phycobilin}

Phycobillins are group of compounds that act as a chromophore in the light harvesting chromoproteins. It is having an open chain tetrapyrrole structure. These phycobillins are efficient absorbers of colours like red, blue, orange etc. There are different types of phycobillins:

a) Phycoerythrobilin-produces red

b) Phycourobilin-produces orange

c) Phycoviolobilin

d) Phycocyanobilin-produces blue [48]

\section{Applications of porphysome nanovesicles}

Porphysomes have a wide range of clinical applications. Some of these are illustrated.

\section{1) Pancreatic cancer therapy}

Pancreatic cancer is one a kind of cancer where the local disease control is of greater concern. It is a disease in which cancerous cells develop in the tissues of pancreas. The pancreas is a gland located behind the stomach and in front of the spine. Pancreatic tumor is often undiagnosed until there is an appearance of clinical symptoms and signs of advanced disease [49]. The primary tumor of pancreatic cancer cannot be surgically resectioned in all the patients. Photothermal therapy can be used for the focal ablation of pancreatic tumor. Photothermal sensitizers in helps in the efficient conversion of light energy to heat and the thermal destruction of the tumor cells with improved special confinement. By fluorescence intensity measurements the accumulation and distribution of tumor can be evaluated. These studies give information regarding the influence of heat on thermal therapy for pancreatic tumors [50].

\section{2) Selective destruction of prostate tumor using MRI guided} photothermal therapy

The prostate is a gland that produces a white fluid. It is located below the urinary bladder and near the base of penis. Almost all pancreatic cancers are called adenocarcinomas. They are cancers that start in cells that lines the glands. Most pancreatic cancers start at in the prostate-specific antigen. The prostate-specific antigen, is a protein that is seen inside the prostate gland, and is made by fluid making cells in the gland [51]. Prostate tumors can be almost destructed by using MRI guided photothermal therapy.

The magnetic resonance imaging-guided laser therapy showed a response in phase 1 trial of low/intermediate-risk localized prostate cancer. This treatment lacked the tumor selectivity as well as had low efficiency of thermal heat generation. In order to resolve this drawback, porphysome nanoparticles have been used to treat localized prostate cancer, having high efficiency and tumor selectivity. Porphysome enabled photothermal eradication of tumor cells without damaging adjacent organs and tissues [52-54]. Porphyrin molecules are fluorescent, naturally. By using a light at one wavelength $(\sim 410$ $\mathrm{nm})$, these molecules are excited. This excitation leads to the reemission of light of higher wavelength $(\sim 600 \mathrm{~nm})$. A charge-coupled device can be used to detect this emitted light. The porphysomes accumulate in the prostate tumors and create an optical signal. This signal is used by surgeons to see the tumor margins during surgery. By making these margins visible, it possible to either removes or ablate tumors by sparing the normal surrounding tissues.

\section{3) Treatment of central serous retinopathy}

Central serous retinopathy (CSR), is an ophthalmic disease which leads to vision loss. It is also called as central serous chorioretinopathy (CSCR) and central serous choroidopathy. It usually affects one of the eye. This disease is often characterized by fluid leakage under the retina. It results from the buildup of fluid under retina. This leads to visual impairment. It is also characterized by a serous detachment of neurosensory retina $[55,56]$.

This disorder can be effectively cured with the intake of a drug named Verteporfin. Verteporfin is a benzoporphyrin derivative. It is used for photodymanic therapy (PDT) as a photosensitizer. It eliminates the abnormal blood vessels in the eye. Verteporfin accumulates in these blood vessels and gets stimulated by nonthermal red light in the presence of oxygen, leads to the formation of reactive oxygen radicals, resulting in the local endothelial damage and blockage of blood vessels [57].

\section{4) Flourescence guided transbronchial photothermal therapy}

Flourescence guided transbronchial photothermal therapy for the treatment of peripheral lung cancer was discovered enabling the porphysomes fig. 9 [58]. The efficiency of transbronchial photothermal therapy was enhanced with the incorporation of porphysomes. Porphysomes enabled the selective and efficient ablation of the tumor in the rabbit model. Lung cancer is divided into small cell lung cancers and non-small cell lung cancers $[59,60]$.

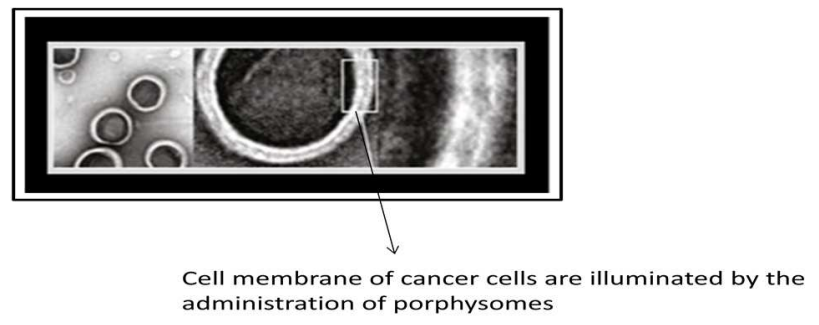

Fig. 9: Detection of cancer cells by porphysomes through fluorescence therapy

\section{5) Ovarian cancer treatment with porphysomes}

Ovarian cancer is the sixth most common cancer among women. It is one of the most dangerous gynaecologic malignancy in many countries including India [61]. Ovarian cancer is defined as the growth of cancerous cells in the ovary. The porphysomes are largely used for the treatment of ovarian cancers in the recent era. 


\section{CONCLUSION}

Vesicular drug delivery system plays an important role in selective targeting of various types of drugs. Among them, Porphysomes are an emerging as well as a more preferred drug delivery system. It can be used as an alternative drug delivery system for liposomes as it presents a similar structure as that of liposomes. It also possess a lot of advantages over liposomes and other systems. A review on liposomes by Nair et al., explains the properties of liposomal drug delivery sytems. From this review, almost all the characters of these carriers are well understood and that helps to compare these characters with the porphysomal drug delivery sytems.

Porphysomal drug delivery systems are having a wide range of applications in the field of photothermal therapy, photoacoustic imaging, radiolabeling etc. It is having very high bioavailability as compared to other forms of drug delivery systems.

\section{ACKNOWLEDGEMENT}

We are immensely obliged and thankful to Dr. Sabitha M., Principal, Amrita School of Pharmacy, Amrita Vishwa Vidyapeetham. We also extend our gratitude to The Department of Pharmaceutics for catering legitimate facilities for carrying out the research work.

\section{AUTHORS CONTRIBUTIONS}

All the author have contributed equally

\section{CONFLICT OF INTERESTS}

\section{Declared none}

\section{REFERENCES}

1. Seema MJ, Pournima M, Manisha K, Vilasrao K. Novel drug delivery system: an overview. J Appl Pharm Sci 2011;22313354.

2. Vijaya SB, Mrudula T, Pavankumar V. Imperitive note on novel drug delivery system. J Nanomed Nanotechnol 2011;2:125.

3. Kumar V, Sharma A, Sharma A, Joshi G, Dhillon V. Recent advances in ndds(novel drug delivery system) for delivery of antihypertensive drugs. Int J Drug Dev Res 2011;3:252-9.

4. Gaurav T, Ruchi T, Birendra S, Bhati L, Pandey S, Pandey P, et al. Drug delivery systems: an updated review. Int J Pharm Invest 2012;2:2-11.

5. Bhargav E, Madhuri N, Ramesh K, Anand M, Ravi V. Targeted drug delivery-a review. World J Pharm Pharm Sci 2013;3:150-69.

6. Reddy PD, Swarnalatha D. Recent advances in novel drug delivery systems. Int J Pharm Res 2010;2:2025-27.

7. Charman WN, Chan HK, Finnin BC, Charman SA. Drug delivery: a key factor in realising the full therapeutic potential of drugs. Drug Dev Res 1999;46:316-27.

8. Manivannan R, Kugalur GP. Recent advances in novel drug delivery systems. Int J Res Ayurveda Pharm 2010;1:316-26.

9. Nuno M, Christiane D, Catarina PR. Recent advances in drug delivery systems. J Biomater Nanobiotechnol 2011;2:510-26.

10. Bassam ARH. Overview on drug delivery system. Pharma Anal Acta 2012;3:137.

11. Aswathy SN, Vidya KM, Saranya TR, Sreeja CN. Emulsomes: a novel liposomal formulation for sustained drug delivery. Int Res J Pharm Appl Sci 2013;3:192-6.

12. Elizabeth H, Gang Z. Porphysome nanotechnology: a paradigm shift in lipid-based supramolecular structures. Nano Today 2014;9:212-22.

13. Mastrobattista E, Koning GA, Storm G. Immunoliposomes for targeted delivery antitumour drugs. Adv Drug Delivery Rev 1999;40:103-27.

14. Shefrin S, Sreelaxmi CS, Vishnu V, Sreeja CN. Enzymosomes: a rising effectual tool for targeted drug delivery system. Int J Appl Pharm 2017;9:1-9.

15. Archana P, Satyendra M, Alka T, Krishna M. Targeted drug delivery (site-specific drug delivery). J Sci Ind Res 2004; 63:230-47.

16. Kenneth KN, Misa T, Cheng CS, Gang Z. Self-sensing porphysomes for fluorescence-guided photothermal therapy. Bioconjug Chem 2015;26:345-51.
17. Vijey A, Samuel GG. Structure-affinity relationship and characterization of benzoporphyrins as potent inhibitors of YAP oncoprotein in silico experiments. Int J Pharm Pharm Sci 2015;7:278-84.

18. Shah H, Patel J. Bicelle: a lipid nanostructure for transdermal delivery. J Crit Rev 2016;3:17-22.

19. Soumya S, Doney AB, Sabitha M. Current trends in lipid-based delivery systems and its applications in drug delivery. Asian J Pharm Clin Res 2012;4:4-9.

20. Upendra B, Sindhu D, Nagavendra K, Wahid K. Liposomal formulations in clinical use: an updated review. Pharmaceutics 2017;9:12.

21. Gert F, Torsten K, Armin W, Alfred B. Phospholipids and lipidbased formulations in oral drug delivery. Pharm Res 2010;27:1469-86.

22. Jing L, Xuling W, Ting Z, Chunling W, Zhenjun H, Xiang L, Yihui D. A review on phospholipids and their main applications in drug delivery systems. Am J Plant Sci 2015;10:81-98.

23. Jindal K. Niosomes as a potential carrier system: a review. Int J Pharm Chem Biol Sci 2015;5:947-59.

24. Ravi B, Sreekanth N, Bhargavi S, Babu RC. Vesicular drug delivery system-an overview. Res J Pharm Biol Chem Sci 2014;4:462-74.

25. Sunil K. Vesicular drug delivery systems: a novel approach for drug targeting. Int J Drug Delivery 2013;5:121-30.

26. Cheng SJ, Marta O, Liyang C, Brian CW. Nanoparticle-enabled selective destruction of the prostate tumor using MRI-guided focal photothermal therapy. Prostate 2016;76:1169-89.

27. Lili Z, Hong W, Bin H, Lijuan Z. Current approaches of photothermal therapy in treating cancer metastasis with nanotherapeutics. Theratonics 2016;6:762-74.

28. Jilong $\mathrm{W}$, Jingjing $\mathrm{Q}$. A review of organic nanomaterials in photothermal cancer therapy. Cancer Res Front 2016;2:67-84.

29. Patrizia A, Kristian B, Keith AC, Thomas HF. Photodynamic therapy of cancer: an update. CA Cancer J Clin 2011;61:250-81.

30. Xianghong Z, Tang L, Zhihong L, Xiangsheng Z. Progress of photodynamic therapy applications in the treatment of musculoskeletal sarcoma (review). Oncol Lett 2014;8:1403-8.

31. Agostinis P, berg K, Cengel KA, Foster TH. Photodynamic therapy of cancer: an update. CA Cancer J Cin 2011;61:250-81.

32. Januz B, Joanna P, Joanna Z. Nanoparticles of titanium and zinc oxides as a novel agent in tumor treatment: a review. Nanoscale Res Lett 2017;12:1-15.

33. Judith W, Paul CB, Sarah EB. Contrast agents for molecular photoacoustic imaging. Nat Methods 2016;13:639-50.

34. Minghua $X$, Lihong V. Photoacoustic imaging in biomedicine. Rev Sci Instrum 2006;77:4110.

35. Langstrom T, Kihlberg Bergstrom. Structure of 2Benzoylamino-5-diethylamino-1,6,6alambda4-triselena-3,4diazapentalene. Acta Chem Scandinavia 1999;53:651.

36. Saraswathi M, Umasankar K, Jayachandra RP. A review on liposomes. Int J Res Pharm Nanol Sci 2014;3:159-69.

37. Rutuja RD, Snehal VG, Megha KB, Priyanka AA. A review on liposomes. World J Pharm Pharm Sci 2016;5:506-17.

38. Bhama SK, Rakhi K, Lakshmi P, Deepa TV, Sreeja CN. Formulation and evaluation of niosomal suspension of cefixime. Asian J Pharm Clin Res 2017;10:194-201.

39. Gnaneswaran S, Kuberan D, Vinodhini VM, Swamy RSV, Ebenezer WW, Kumar JS. Fasting plasma glucose and glycolated haemoglobin in prediction of diabetic retinopathyin rural population. Int J Pharm Clin Res 2014;6:40-5.

40. Alan NS. Hemoglobin research and the origins of molecular medicines. Blood 2008;112:3927-38.

41. Stefan H, Bernhard K. Chlorophyll breakdown in higher plants. Biochim Biophys Acta 2011;1807:977-88.

42. Aris H, Su L, Ronald H, Michael KD. Chlorophyll extract from microalgae: a review on the process engineering aspects 2010:1-10. http://dx.doi.org/10.1155/2010/391632

43. Galina PB, Ekaterina AS. Myoglobin and mitochondria: how does the "oxygen store" work? J Phys Chem Biophys 2013;3:1-7.

44. George AO, Daniel JG. Myoglobin: an essential hemoprotein in striated muscle. J Exp Biol 2004;207:3441-6.

45. Shane BK, Pradeep PA. Regualation of gene expression. J Exp Biol 2010;213:2741-7. 
46. Caroline PB, Henrik JA. Myoglobin-induced lipid oxidation-a review. J Agric Food Chem 2002;50:3887-97.

47. Nelson DL, Cox M. Lehninger, principles of biochemistry. $3^{\text {rd }}$ ed. Worth Publishing: New York; 2000.

48. Samuel IB. Biosynthesis of phycobilins. Chem Rev 1993; 93:785-802.

49. Rosella S, Federica B, Agostino P, Isabella C. Current therapeutic strategies for advanced pancreatic cancer: a review for clinicians. World J Clin Oncol 2016;7:27-43.

50. Mac Laughlin CM, Ding L, Jin C, Cao P, Siddiqui I, Hwang DM, et al. Porphysome nanoparticles for enhanced photothermal therapy in a patient-derived orthotopic pancreas xenograft cancer model: a pilot study. J Biomed Opt 2016;21:84002.

51. Bozeman CB, Carver BS, Caldito G, Venable DD. Prostate cancer in patient with an abnormal digital rectal examination and serum prostate-specific antigen less than $4.0 \mathrm{ng} / \mathrm{ml}$. Urology 2005;66:803-73.

52. Litwin MS, Tan HJ. The diagnosis and treatment of prostate cancer: a review. JAMA 2017;317:2532-42.

53. Jin CS, Overchuk M, Cui L, Wilson BC, Bristow RG, Chen J, et al. Nanoparticle-enabled selective destruction of the prostate tumor using MRI-guided focal photothermal therapy. Prostate 2016;76:1169-81.

54. Maya S, Sabitha M, Shanthikumar VN, Jayakumar R. Phytomedicine-loaded polymeric nanomedicines: potential cancer therapeutics. Adv Polym Sci 2013;254:203-39.

55. Iacono P, Battaglia PM, Falcomata B, Bandello F. Central serous chorioretinopathy treatments: a mini review. Ophthalmic Res 2016;55:76-83.

56. Alenjandra D, Alexandre M, Ali D, Elodie B. Central serous chorioretinopathy: Recent findings and new physiopathology hypothesis. Prog Retinal Eye Res 2015;48:82-118.

57. Marwan AA. Advances in the treatment of central serous retinopathy. Saudi J Ophthalmol 2015;29:278-86.

58. Alexandros GS. Nanotechnology-enabled thernostics of lung cancer. Cancer Res 2016;76:5870-80.

59. Kelly ML, Timothy FM. Lung cancer: diagnosis, treatment, principles and screening. Am Fam Physician 2015;91:250-6.

60. Roy SH, John VH, Scott ML. Lung cancer. N Eng J Med 2008;359:1367-80.

61. Marilena VI, Rosa V, Gianpiero DL, Valentina d. Micro RNA signatures in human ovarian cancer. Cancer Res 2007; 67:8699-707. 